Collaboration between clinicians, geneticists, and basic scientists as well as the clever use of mouse models will certainly help address some of these questions and enhance our understanding of IRF6- and p63-related diseases, the ultimate beneficiaries being the afflicted patients and their families.

\section{Acknowledgments}

I am grateful to Anders Linde for preparing the figures and to Sara Rizell for providing the clinical photo. The author is supported by the Swedish Research Council-Medicine (grants 15181 and 20614), the Institute of Odontology at Sahlgrenska Academy, and the Thuréus Foundation.

Address correspondence to: Amel GritliLinde, Department of Oral Biochemistry, Sahlgrenska Academy, University of Gothenburg, Medicinaregatan 12F, SE-41390 Göteborg, Sweden. Phone: 46.31.786.3386; Fax: 46.31.418122; E-mail: amel@odontologi.gu.se.

\footnotetext{
1. Rinne T, Bruner HG, van Bokhoven H. P63-associated disorders. Cell Cycle. 2007;6(3):262-268.
}

2. Kondo S, et al. Mutations in IRF6 cause van der Woude and popliteal pterygium syndromes. Nat Genet. 2002;32(2):285-289.

3. Gritli-Linde A. The etiopathogenesis of cleft lip and cleft palate: Usefulness and caveats of mouse models. Curr Top Dev Biol. 2008;84:37-138.

4. Mikkola ML. P63 in skin appendage development. Cell Cycle. 2007;6(3):285-290.

5. Thomason HA, Dixon MJ, Dixon J. Facial clefting in Tp63 deficient mice result from altered Bmp4, Fgf8 and Shh signaling. Dev Biol. 2008;321(1):273-282.

6. Ingraham CR, et al. Abnormal skin, limb and craniofacial morphogenesis in mice deficient for interferon regulatory factor 6 (Irf6). Nat Genet. 2006;38(11):1335-1340.

7. Richardson RJ, et al. Irf6 is a key determinant of the keratinocyte proliferation-differentiation switch. Nat Genet. 2006;38(11):1329-1334

8. Bailey CM, Hendrix MJC. IRF6 in development and disease: A mediator of quiescence and differentiation. Cell Cycle. 2008;7(13):1925-1930.

9. Moretti F, et al. A regulatory feedback loop involving $\mathrm{p} 63$ and IRF 6 links the pathogenesis of 2 genetically different human ectodermal dysplasias. J Clin Invest. 2010;120(5):1570-1577.

10. Thomason HA, et al. Cooperation between the transcription factors p 63 and IRF6 is essential to prevent cleft palate in mice. J Clin Invest. 2010;120(5):1561-1569.

11. Rahimov F, et al. Disruption of an AP- $2 \alpha$ binding site in an IRF6 enhancer is strongly associated with cleft lip. Nat Genet. 2008;40(11):1341-1347.

12. Richardson RJ, Dixon J, Jiang R, Dixon MJ. Integration of IRF6 and Jagged 2 signalling is essential for controlling palatal adhesion and fusion competence. Hum Mol Genet. 2009;18(14):2632-2642.

13. Xu X, et al. Ectodermal Smad4 and p38 MAPK are functionally redundant in mediating TGF- $\beta /$ BMP signaling during tooth and palate development. Dev Cell. 2008;15(2):322-329.

14. Westfall MD, Mays DJ, Sniezek JC, Pietenpol JA. The $\Delta \mathrm{Np} 63 \alpha$ phosphoprotein binds the $\mathrm{p} 21$ and $14-3-3 \sigma$ promoters in vivo and has transcriptional repressor activity that is reduced by Hay-Wells syndrome-derived mutations. Mol Cell Biol. 2003;23(7):2264-2276.

15. Adorno $M$, et al. A mutant-p53/Smad complex opposes p 63 to empower Tgf $\beta$-induced metastasis. Cell. 2009;137(1):87-98.

16. Ghioni $\mathrm{P}$, et al. The protein stability and transcriptional activity of $\mathrm{p} 63 \alpha$ are regulated by SUMO-1 conjugation. Cell Cycle. 2005;4(1):183-190.

17. Rossi M, et al. The E3 ubiquitin ligase Itch controls the protein stability of p63. Proc Natl Acad Sci US A. 2006;103(34):12753-12758

18. Li Y, Zhou Z, Chen C. WW domain-containing E3 ubiquitin protein ligase 1 targets $\mathrm{p} 63$ transcription factor for ubiquitin-mediated proteasomal degradation and regulates apoptosis. Cell Death Differ. 2008;15(12):1941-1951.

19. Little HJ, et al. Missense mutations that cause van der Woude syndrome and popliteal pterygium syndrome affect the DNA-binding and transcriptional activation functions of IRF6. Hum Mol Genet. 2009;18(3):535-545.

20. Fomenkov A, et al. RACK1 and Stratifin target $\Delta \mathrm{Np} 63 \alpha$ for a proteasome degradation in head and neck squamous cell carcinoma cells upon DNA damage. Cell Cycle. 2004;3(10):1285-1295.

\title{
Cryptococci at the brain gate: break and enter or use a Trojan horse?
}

Arturo Casadevall

Department of Microbiology and Immunology and Medicine, Albert Einstein College of Medicine, New York, New York, USA.

\begin{abstract}
The mechanism by which Cryptococcus neoformans invades the central nervous system is fundamental for understanding pathogenesis because cryptococcosis commonly presents as meningoencephalitis. There is evidence for both direct invasion of the endothelial cells lining the brain vasculature and a "Trojan horse" mechanism whereby cryptococci enter the central nervous system after macrophage ingestion. However, in this issue of the JCI, Shi et al. use intravital microscopy to reveal that brain invasion by C. neoformans follows a capillary microembolic event. They find that after suddenly stopping in brain capillaries, cryptococci cross into the central nervous system in a process that is urease dependent, requires viability, and involves cellular deformation. This observation provides evidence for direct brain invasion by C. neoformans, but a consideration of all the currently available evidence suggests a role for both direct and phagocyte-associated invasion. Hence, the remarkable neurotropism of $C$. neoformans may have more than one mechanism.
\end{abstract}

Cryptococcus neoformans is a soil-dwelling fungus that emerged in the late twentieth century as a major human pathogen

Conflict of interest: Arturo Casadevall is a consultant and holds stock options in Pain Therapeutics Inc.

Citation for this article: J Clin Invest. 2010;

120(5):1389-1392. doi:10.1172/JCI42949. because of its propensity to cause lethal meningoencephalitis. The burden of cryptococcosis is estimated to approach one million cases per year, with a mortality that exceeds that from tuberculosis (1). C. neoformans is acquired by inhalation of dehydrated cells or spores (2). Serologic surveys indicate a high prevalence of human infection, which is likely to be first acquired in childhood (3). Although infection is common, disease is rare, and cryptococcosis occurs primarily in hosts with impaired immunity, such as patients with AIDS, organ transplant recipients, and those treated with immunosuppressive therapies (2). Hence, normal immune responses are believed to control infection in the lung. Extrapulmonary dissemination is therefore invariably associated with disease, with meningoencephalitis being the most common clinical presentation of cryptococcosis. To cause meningoencephalitis, C. neoformans must cross several epithelial and/or endothelial cell layers, first to leave the lung and then to reach the brain. How does a soil-dwelling organism that has no need for animal pathogenesis for survival such as C. neoformans reach the brain to cause meningoencephalitis? In this issue of the JCI, Shi et al. (4) shed new light on this subject by applying 


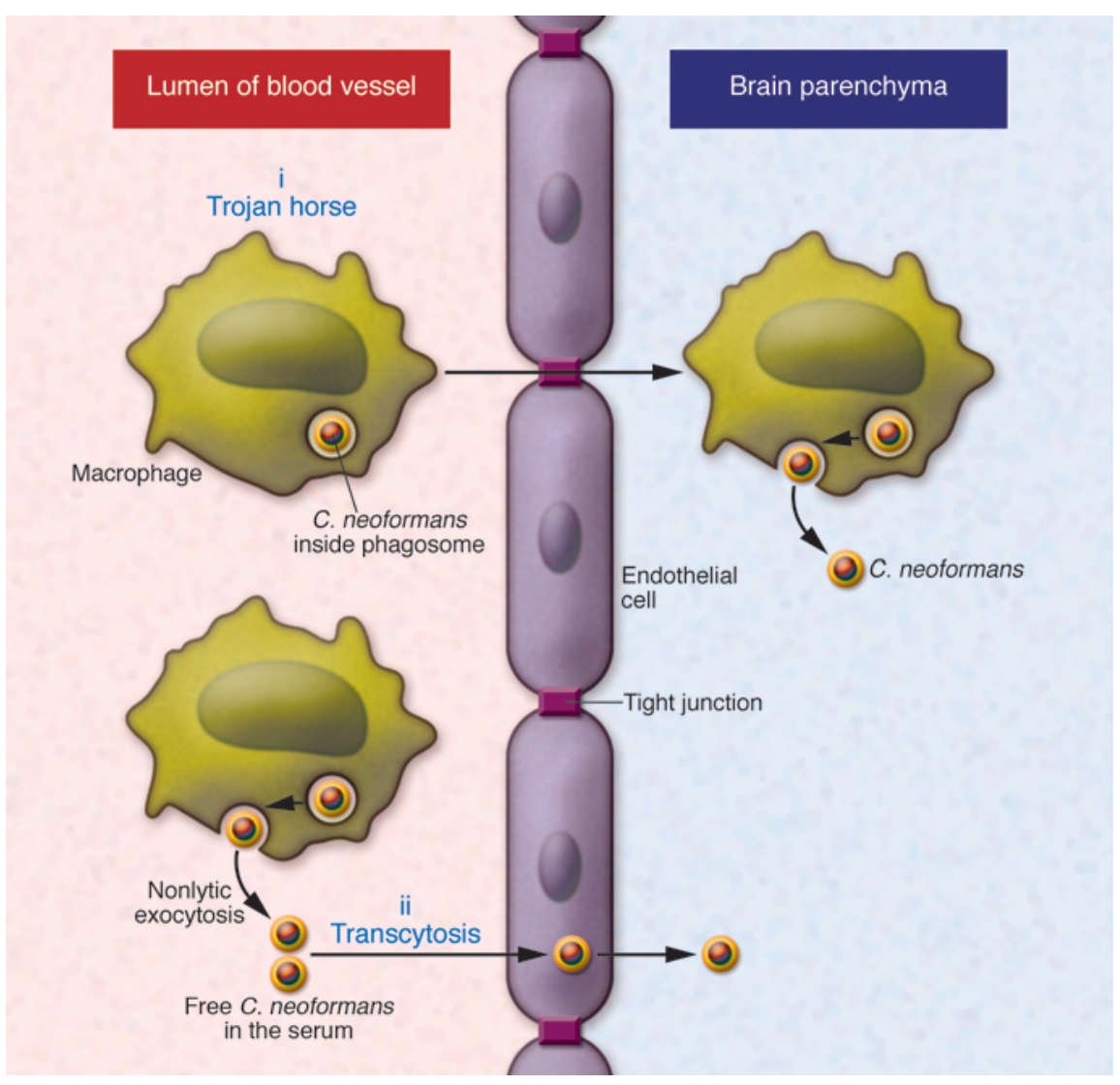

the technique of intravital microscopy to visualize in mice the process of brain invasion by C. neoformans.

\section{Current views of brain invasion}

Although the predisposition of C. neoformans to cause meningoencephalitis has been known for more than a century, the mechanism by which fungal cells invade the central nervous system has remained elusive. In recent years, two competing hypotheses have been proposed for brain invasion (Figure 1). The first mechanism posits a "Trojan horse" approach, whereby fungal cells gain access to the brain by transport in phagocytic cells. The finding that cryptococci in the meningeal vasculature were in close association with phagocytic cells suggested that brain invasion was cell associated (5). Circumstantial evidence for this mechanism is provided by the fact that $C$. neoformans is a facultative intracellular pathogen that can survive in macrophages (6) and that extrapulmonary dissemination appears to be macrophage associated (7-9). Strong experimental evidence for the Trojan horse mechanism came from elegant experiments in which mice were infected with macro- phages containing ingested cryptococci (10). According to this view, fungal cells are phagocytosed first in the blood or the vicinity of the endothelial cells of the brain vasculature and then the phagocytic cell transports them to the parenchyma. The second mechanism posits that naked C. neoformans cells invade the brain by direct transcytosis of endothelial cells lining the brain vasculature (11). This view is supported by in vitro and in vivo observations showing that yeast cells are taken up by endothelial cells and can transit through the cytoplasm to emerge on the other cellular surface (11). It is noteworthy that neither mechanism is exclusive of the other, and in fact, there is some evidence that both can occur simultaneously (10).

\section{Intravital microscopy provides new insights}

In this issue of the JCI, Shi et al. (4) apply the technique of intravital microscopy to visualize in mice the process of brain invasion by C. neoformans. They were able to visualize the brain microvasculature adjacent to the meninges and observe the events preceding, and concurrent with, cryptococcal brain invasion. A major find-

\section{Figure 1}

Mechanisms by which $C$. neoformans has been posited to enter the central nervous system. C. neoformans can travel in blood in either free or phagocytic cell-associated form. Free yeast forms in blood could originate from exocytosis from phagocytic cells or perhaps transmigration from primary infection areas such as the lung. (i) The Trojan horse mechanism. C. neoformans reaches the brain inside an infected phagocytic cell that transports it across from the lumen of a brain capillary to the central nervous system. (ii) Direct transcytosis. $C$. neoformans reaches the brain by direct transmigration of capillary endothelium.

ing of this study is that fungal cells stop suddenly in capillary sites without rolling or tethering, in a manner similar to polystyrene microspheres. The similarity of the arresting process to that exhibited by inert microspheres suggests that the initial brain localization mechanism is mechanical and related to an inability of yeast cells to traverse narrow capillaries. If this is the case, the initial brain infection results from a microembolic event. It was observed that after stopping, C. neoformans cross the capillary wall in a process that requires viability but not replication, is associated with deformation of cell morphology, and is urease dependent, as reported previously (12). Finally, the investigators show that inhibiting urease reduces brain fungal burden, suggesting that this might provide an entirely new approach toward protecting the brain in cryptococcal meningitis. Each of these observations has important repercussions for our understanding of cryptococcal neuropathogenesis.

The finding that the initial brain localization followed sudden arrest in what appears to be a fungal microembolic event suggests that the process may not require specific attachment receptors, as 
has been suggested by in vitro studies (13, 14), although these receptors could still play a role in invasion. If this is the case, the remarkable neurotropism of this fungus may have more to do with mechanical entrapment than with either microbespecific neurotrophic properties or the immune-privileged status of the central nervous system. However, mice inoculated with encapsulated and non-encapsulated strains of C. neoformans manifest comparable brain infection (4), a finding that argues against an effect based solely on diameter, since one might have expected more brain invasion after injection of the encapsulated cells, which are larger by virtue of having a capsule. A caveat in this comparison is that acapsular cells have a propensity to aggregate, and thus it is conceivable that the size difference between encapsulated and acapsular cells was not apparent because the latter involved aggregates of more than one cell. It would be interesting to know whether other yeast cell types such as Candida albicans or Saccharomyces cerevisiae also manifest sudden stopping in the brain vasculature. If these species behave as C. neoformans, then the remarkable neurotropism of cryptococci must have another explanation, since candidemia is not associated with meningoencephalitis. Furthermore, the contribution of cell size and capsule to initial brain localization could also be explored by comparing cells with induced and noninduced capsules. If mechanical trapping is all that is needed for initial invasion, then one might predict that intravenous infection with the larger capsule-induced cells should produce a greater burden of brain infection than infection with smaller cells. This, in turn, would imply a new pathogenic function for the capsule.

Shi et al. (4) also observed C. neoformans directly crossing the capillaries into the brain parenchyma following sudden stopping. Although this observation provides direct in vivo experimental support for the transcytosis model of cryptococcal brain invasion (Figure 1), it is worthwhile pausing and considering the advantages and limitations of this system in the interpretation of the data. The major advantage of intravenous infection is that it allows the researcher to control the time of blood invasion so that real-time visualization of the pathogenic process is possible. However, intravenous infection may or may not reflect the natural mode of brain dissemination. There is widespread consensus in the field that the initial site of human infection is the lung and that extrapulmonary dissemination occurs only in hosts whose immune systems cannot control the fungus in the lung. Macrophages appear to be critically important cells in determining the outcome of infection, such that resistance to infection has been correlated with their ability to restrict intracellular fungal replication (15). Recently, indirect evidence has emerged that extrapulmonary dissemination is cell associated, with fungal cells leaving the lung in phagocytic cells, possibly macrophages. If this is the case, then the intravenous model, whereby fungal cells are injected directly into the bloodstream, may not seem that relevant to the natural processes responsible for meningoencephalitis. However, even if extrapulmonary dissemination is cell associated, the finding that C. neoformans is capable of nonlytic exocytosis of infected cells $(16,17)$ suggests that infected cells can release free C. neoformans into the circulation that would then be in a situation analogous to that following intravenous infection $(16,17)$. Another caveat in interpreting the results of Shi et al. (4) is that cryptococci are normally ingested by macrophages in chronic pulmonary infection, and the current experiment was done in mice infected intravenously such that there may not have been sufficient time for phagocytic cells to ingest them. Repeating the experiments performed by Shi et al. (4) using a pulmonary route of infection with $C$. neoformans might provide data to resolve the direct versus Trojan horse invasion scenarios, although such studies could be technically demanding, since the researcher would not be able to control the timing of extrapulmonary dissemination and know when to monitor the brain vasculature. However, perhaps this experimental hurdle could be bypassed by injecting macrophages containing ingested cryptococci and observing their interaction with the brain vasculature.

\section{Fungal activities promote invasion}

In the study by Shi et al. (4), there are other pearls worthy of comment. First, the observation that the fungus changes its shape from spherical to ovoid during the process of brain invasion, which raises the possibility that the cryptococcal cell skeleton and cell wall are altered to facilitate this process. This observation is fascinating, because it implies that C. neoformans, like other fungi, is capable of morphological change, in a process that gives it access to another niche and has distant echoes in the morphological changes observed during tissue invasion by dimorphic fungi and C. albicans. Hence, shape changes accompany the changes in capsule structure that have been described during brain invasion (18). Second, the finding that urease activity contributes to brain infection is an observation that merits intensive study to ascertain the mechanisms involved. Third, the observation that pharmacologic inhibition of urease activity reduces brain infection provides a new precedent for protecting the brain. Although such an approach is likely to have limited usefulness in most cases of human cryptococcosis, where the majority of patients present with meningoencephalitis, and therefore already have substantial brain infection, the notion that enzymatic inhibition translates into reduced neural invasion does open new areas of investigation that may be exploited in the future. For example, if the effect of urease is extracellular, it might be possible to protect the brain parenchyma against cryptococcal infection by raising urease-specific neutralizing antibody responses.

\section{Transmigration and/or Trojan horses}

The article by Shi et al. (4) is a major contribution to the cryptococcal field because it applies a new technique to the study of the pathogenesis of C. neoformans meningoencephalitis that gives fresh new insights into the mechanisms involved and poses many new questions for future studies. As to the question of whether brain invasion is the result of direct transmigration or occurs via a Trojan horse, while the data generated by Shi et al. (4) support the former, the totality of the available evidence strongly suggests that both mechanisms occur. Debates as to the relative importance of each mechanism are not helpful, since it is likely that one or the other predominates depending on the host and/or the conditions of the experiment. Instead, it is fortunate that the cryptococcal field has at least two different mechanisms for brain invasion to study, each fascinating on its own, and each providing an abundance of questions for future study.

\section{Acknowledgments}

A. Casadevall is supported by NIH grants HL059842, AI033774, AI033142, and AI052733.

Address correspondence to: Arturo Casadevall, Department of Microbiology and Immunology, 1300 Morris Park Ave., Bronx, New York 10461, USA. Phone: 718.430.3665; Fax: 718.430.8711; E-mail: Arturo.casadevall@einstein.yu.edu. 
1. Park BJ, Wannemuehler KA, Marston BJ, Goven der N, Pappas PG, Chiller TM. Estimation of the current global burden of cryptococcal meningitis among persons living with HIV/AIDS. AIDS 2009;23(4):525-530

2. Casadevall A, Perfect JR. Cryptococcus neoformans. Washington, DC: American Society for Microbiology; 1998 .

3. Goldman DL, et al. Serologic evidence for Cryptococcus infection in early childhood. Pediatrics. 2001;107(5):E66.

4. Shi M, et al. Real-time imaging of trapping and urease-dependent transmigration of Cryptococcus neoformans in mouse brain. J Clin Invest. 2010; 120(5):1683-1693

5. Chretien F, Lortholary O, Kansau I, Neuville S, Gray F, Dromer F. Pathogenesis of cerebral Cryptococcus neoformans infection after fungemia. J Infect Dis. 2002;186(4):522-530.

6. Feldmesser M, Kress Y, Novikoff P, Casadevall A. Cryptococcus neoformans is a facultative intracellular pathogen in murine pulmonary infection. Infect Immun. 2000;68(7):4225-4237.

7. Shea JM, Kechichian TB, Luberto C, Del Poeta M The cryptococcal enzyme inositol phosphosphin- golipid-phospholipase $\mathrm{C}$ confers resistance to the antifungal effects of macrophages and promotes fungal dissemination to the central nervous system. Infect Immun. 2006;74(10):5977-5988.

8. Kechichian TB, Shea J, Del PM. Depletion of alveolar macrophages decreases the dissemination of a glucosylceramide-deficient mutant of Cryptococcus neoformans in immunodeficient mice. Infect Immun. 2007;75(10):4792-4798.

9. Santangelo R, et al. Role of extracellular phospholipases and mononuclear phagocytes in dissemination of cryptococcosis in a murine model. Infect Immun. 2004;72(4):2229-2239.

10. Charlier C, Nielsen K, Daou S, Brigitte M, Chretien $\mathrm{F}$, Dromer F. Evidence of a role for monocytes in dissemination and brain invasion by Cryptococcus neoformans. Infect Immun. 2009;77(1):120-127.

11. Chang YC, et al. Cryptococcal yeast cells invade the central nervous system via transcellular penetration of the blood-brain barrier. Infect Immun. 2004;72(9):4985-4995

12. Olszewski MA, et al. Urease expression by Cryptococcus neoformans promotes microvascular sequestration, thereby enhancing central nervous system invasion. Am J Pathol. 2004;164(5):1761-1771.
13. Jong A, et al. Involvement of human CD44 during Cryptococcus neoformans infection of brain microvascular endothelial cells. Cell Microbiol. 2008;10(6):1313-1326.

14. Jong A, et al. Invasion of Cryptococcus neoformans into human brain microvascular endothelial cells requires protein kinase $\mathrm{C}$-alpha activation. Cell Microbiol. 2008;10(9):1854-1865.

15. Shao X, Mednick A, Alvarez M, van Rooijen N, Casadevall A, Goldman DL. An innate immune system cell is a major determinant of species-related susceptibility differences to fungal pneumonia. Immunol. 2005;175(5):3244-3251.

16. Alvarez M, Casadevall A. Phagosome fusion and extrusion, and host cell survival following Cryptococcus neoformans phagocytosis by macrophages. Curr Biol. 2006;16(21):2161-2165.

17. Ma H, Croudace JE, Lammas DA, May RC. Expulsion of live pathogenic yeast by macrophages. Curr Biol. 2006;16(21):2156-2160.

18. Charlier C, Chretien F, Baudrimont M, Mordelet E, Lortholary O, Dromer F. Capsule structure changes associated with Cryptococcus neoformans crossing of the blood-brain barrier. Am J Pathol. $2005 ; 166(2): 421-432$

\title{
Caveat mTOR: aberrant signaling disrupts corticogenesis
}

\author{
Lucy R. Osborne
}

Department of Medicine and Department of Molecular Genetics, University of Toronto, Toronto, Ontario, Canada.

\begin{abstract}
The mammalian target of rapamycin (mTOR) signaling pathway is activated in several disorders associated with benign tumors and malformations of the cerebral cortex. In this issue of the JCI, Orlova et al. have now definitively added another disorder to this group by demonstrating that activation of mTOR signaling is associated with polyhydramnios, megalencephaly, and symptomatic epilepsy syndrome (PMSE), which is characterized by severe intractable epilepsy and megalencephaly. PMSE is caused by lack of the pseudokinase STE20-related kinase adaptor $\alpha($ STRAD $\alpha)$, and Orlova et al. show that reduction of STRAD $\alpha$ levels during corticogenesis in the mouse results in a cellular phenotype and neuronal migration defects similar to those observed in patients with PMSE, clearly demonstrating a pivotal role for STRAD $\alpha$ in cell polarity and growth. This study helps pave the way for possible therapeutic intervention with rapamycin to control the epilepsy and learning disabilities associated with this disorder.
\end{abstract}

Development of the human cortex is a complex and finely orchestrated process. The layers of the developing neocortex are generated through neuronal migration, whereby neurons travel from their place of origin in the proliferating ventricular zone to their final position in the brain. This cortical lamination is generated from the inside out, with the neurons that contribute to each layer traveling past the existing

Conflict of interest: The author has declared that no conflict of interest exists.

Citation for this article: J Clin Invest. 2010; 120(5):1392-1395. doi:10.1172/JCI43030. layers; perturbation of this carefully controlled neuronal migration can result in heterotopia (neurons in the wrong place) and dysplasia (disorganization of the normal structure of the cortex) that disrupt the normal cortical circuitry. Imbalance between excitatory and inhibitory systems in the cortex can lead to spontaneous electrical discharge with catastrophic consequences, often in the form of intractable or medication-resistant epilepsy and severe intellectual disability (1).

Polyhydramnios, megalencephaly, and symptomatic epilepsy syndrome (PMSE), recently described in an Old Order Men- nonite population, is associated with craniofacial dysmorphism (large forehead, widely spaced eyes, and large mouth), an abnormally large brain, and severe, earlyonset intractable epilepsy (2). PMSE in this kindred is caused by homozygous deletion of a portion of the STE20-related kinase adaptor $\alpha$ gene (STRADA; encoding STRAD $\alpha$ ) on human chromosome 17 (2). STRAD $\alpha$ normally binds and exports the protein kinase serine/threonine kinase 11 (STK11; also known as LKB1) out of the nucleus, where they bind to MO25 to form a trimeric complex that has an inhibitory effect on mammalian target of rapamycin (mTOR) signaling through the sequential phosphorylation of AMPK and the tuberous sclerosis complex 1 / tuberous sclerosis complex 2 (TSC1/TSC2) complex (Figure 1 and ref. 3). Aberrant activation of the mTOR pathway was identified in the brain of an individual with PMSE by the presence of high levels of phosphorylated ribosomal S6 protein (2), a downstream target of mTOR. This finding is suggestive of a link between the cellular mechanism underlying this disorder and that previously shown to be responsible for a group of syndromes characterized by hamartomas, benign tumors composed of differ- 